\title{
CORPORATE WEBSITE EVALUATION USING PARTIAL LEAST SQUARES PATH MODELLING
}

\author{
PAVOL KRÁL'a,*, PETER LACO ${ }^{\mathrm{a}}$ \\ pavol.kral@umb.sk, peter.laco@umb.sk
}

\begin{abstract}
a Matej Bel University in Banská Bystrica, Faculty of Economics, Department of Quantitative Methods and Information Systems, Tajovského 10, Banská Bystrica, Slovakia
\end{abstract}

\begin{abstract}
Almost all businesses and organizations use the World Wide Web in the form of corporate websites. In today's highly innovative and competitive environment, the level of corporate internet presentations is one of the key success factors. Approaches to corporate website evaluation are very variable. To the best knowledge of the authors, there is no universally accepted and recommended methodology of corporate website evaluation that could be, without any caveats, used in conditions of Slovak small and medium-sized enterprises. The aim of the paper is to fill this gap and propose a methodology tailored to evaluation of websites of Slovak small and medium-sized enterprises. The proposed methodology builds on the previous work of the authors and its core element is a model fitted using partial least squares path modeling.
\end{abstract}

\section{Key words}

internet, internet presentation, web, corporate website, partial least squares path modeling

\section{JEL classification}

D83, M15, O33

\section{Introduction}

Information and communication technologies, specifically websites have had a significant impact on business at a global level. Especially for small and medium enterprises the usage of digital technologies is on one hand a significant opportunity, but on the other hand also a potential threat (Lesáková, 2008). The opportunity comes from the ability of companies to access technologies that were previously only available to large companies and to use these to compete in international markets. Yet the threat emerges from companies losing business by not embracing the opportunities and becoming uncompetitive in increasingly digital and online markets.

As we can see on Figure 1, retail e-commerce sales worldwide from 2014 to 2021 have a steadily growing trend. In 2017, retail e-commerce sales worldwide amounted to 2.3 trillion US dollars and e-retail revenues are projected to grow to 4.88 trillion US dollars in 2021. Another statistics from the same source (http://statista.com, 30-03-2019) shows, that the top 3 online stores' revenue amounted to almost 100 billion US dollars in 2017. Online shopping is one of the most popular online activities worldwide but the usage varies by region. Desktop PCs are still the most popular device for placing online shopping orders but mobile devices, especially smartphones, are catching up.

Figure 2 gives us a more detailed look at the situation in European countries in 2017 as it shows the percentage of total turnover from web sales. We can see that the situation in central European countries like Czech Republic, Poland and Slovakia is approximately the same with share of web sales about $6 \%$ on total sales. It is obvious that the most of sales were realized by own corporate websites or apps. 
Figure 1: Retail e-commerce sales worldwide from 2014 to 2021 (in billions of U.S. dollars)

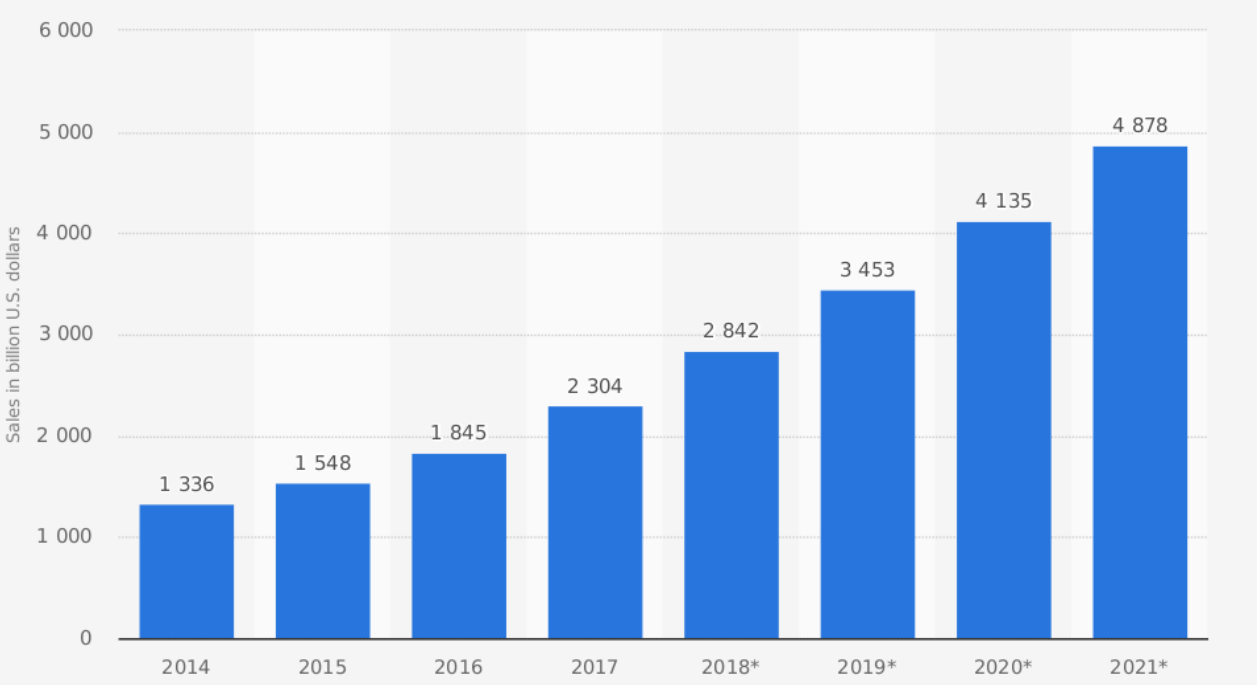

Source: the authors based on data from the Statista portal.

Figure 2: Turnover from web sales broken down by own website or apps and marketplace, 2017 (\% of total turnover)

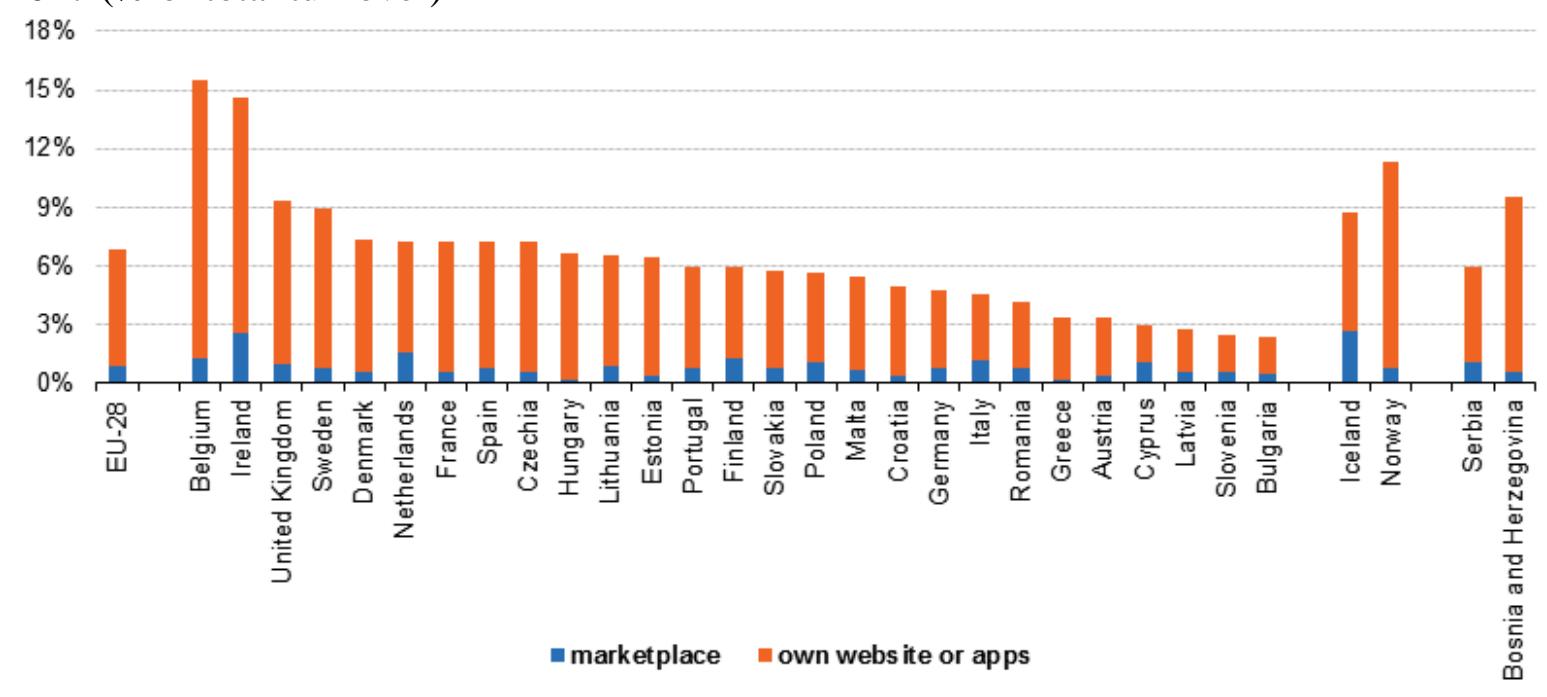

Source: the authors based on data from Eurostat.

Based on statistics showed above, it is clear that the trend of e-commerce growth is indisputable, and the corporate websites plays a crucial role in it. That is why aim of our research is to propose a methodology to evaluate the corporate websites, as they are the most commonly used tool for on-line customers. We are convinced that it might help especially small and medium companies to increase the quality of corporate websites that will lead to increased sales and profit. In our previous research (Kollár et al., 2014; Kollár et al., 2015; Laco and Kallová, 2017) we focused on identification of criteria that are crucial for websites evaluation. We identified these criteria and we verified their importance for web users using questionnaires. In Král and Laco (2018) we introduce a new methodology that was not applied to this field before and we used it on sample data of Slovak SMEs websites. Aim of this paper is to present application of this methodology on much larger sample of data gathered from investigation of websites of top 250 manufacturing companies according to sales. Minor improvements of the model are presented too.

During recent years, different website evaluation approaches have been introduced. These deal mostly with website usability (Alva et al., 2003; Tezza et al., 2011), but also content 
(Robbins and Stylianou, 2003), website accessibility (Leporini and Paterno, 2003), design (Palmer, 2002), functionality, or technical aspects of websites. The complex review of the literature from 1995-2006 was presented by Chiou et al. (2010), who identified that in $41 \%$ of studies technology-oriented factors were used to evaluate websites, such as navigability, accessibility, usability, or information quality. In $12 \%$ of studies marketing factors were used, like advertising, customer service, promotion, order confirmation, or online transaction. In the most of studies (47\%) a combine framework as a mixture of both information system and marketing approaches was used. Chiou et al. (2010) also investigated used research methodologies, that were survey (42\%), experiment (23\%), content analysis (17\%), case study $(10 \%)$, automatic evaluation (5\%) and concept development (3\%).

\section{Methodology}

We evaluate websites via scoring derived using Partial Least Squares Path Modeling (PLSPM) methodology presented (Sanchez, 2013) which is a component-based approach to the latent variable modeling. The goal is to generate latent variable scores (factor scores) representing some general concepts, e.g., quality, functionality, usability, having at hand values of manifest, i.e. directly measurable, variables connected to the assumed latent variables.

A model in PLSPM methodology consist of two parts - an inner one and an outer one. The inner part describes relationship among assumed latent variables, the outer part connects latent variables and the corresponding manifest variables. We aim for latent scores which are as much correlated to each as possible and the most representative of their corresponding manifest variables.

In the paper, we assume that manifest variables simple reflects (are caused by) latent variables, i.e. we assume so-called reflexive manifest variables where one set of manifest variables corresponds to each latent variable.

Formally, following Sanchez (2013), we assume a data set X consisting of n observations and $\mathrm{p}$ variables which can be divided in $J$ mutually exclusive blocks $\mathrm{X}_{1}, \mathrm{X}_{2}, \ldots, \mathrm{X}_{J}$ and each of these blocks is associated with a latent variable $L V_{\mathrm{j}}$. We denote estimation of this latent variable by $Y_{j}$. For the inner model we assume for at

$$
L V_{j}=\beta_{0}+\sum_{i \rightarrow j} \beta_{j i} L V_{i}+\varepsilon_{j},
$$

where latent variables $L V_{\mathrm{i}}$ predict $L V_{\mathrm{j}}, \beta_{j i}$ are path coefficients and $\operatorname{cov}\left(L V_{j}, \varepsilon_{j}\right)=0$.

For the outer model it holds that

$$
X_{j k}=\lambda_{0 j k}+\lambda_{j k} L V_{j}+\varepsilon_{k},
$$

where $\lambda_{j k}$ are called loadings and $X_{j k}$, denotes the $k$-th manifest variable in the $j$-th block.

Scores $Y_{j}$ corresponding to a latent variable $L V_{j}$ are then estimated by

$$
Y_{j}=\sum_{k} w_{j k} X_{j k}
$$

where $w_{j k}$ denotes an outer weight corresponding to a manifest variable $X_{j k}$.

The outer weights $w_{j k}$ are determined as follows. First, we assign to each variable $X_{j k}$ a weight equals one. Then these weights are transformed to $\tilde{w}_{j k}$ such that the resulting scores have a unit variance. Then it holds that

$$
Y_{j}= \pm f_{j} \sum_{k} \tilde{w}_{j k} X_{j k}
$$


where $f_{j}$ a number standardizing $Y_{j}$ and we choose the sign for each weight such that the majority of $X_{j k}$ is positively correlated with the resulting $Y_{j}$.

From the computational point of view, PLSPM model fitting is an iterative process consisting of four steps:

1. determination of the outer weights,

2. computation of the latent variable scores,

3. estimation of the path coefficients,

4. estimation of the loadings.

\subsection{Model and data}

We adopted a simple path model (Figure 3) presented in (Král' and Laco, 2018), where we replaced the Error.warnings variable used in the original model by Broken.links variable.

Figure 3: Websites evaluation model

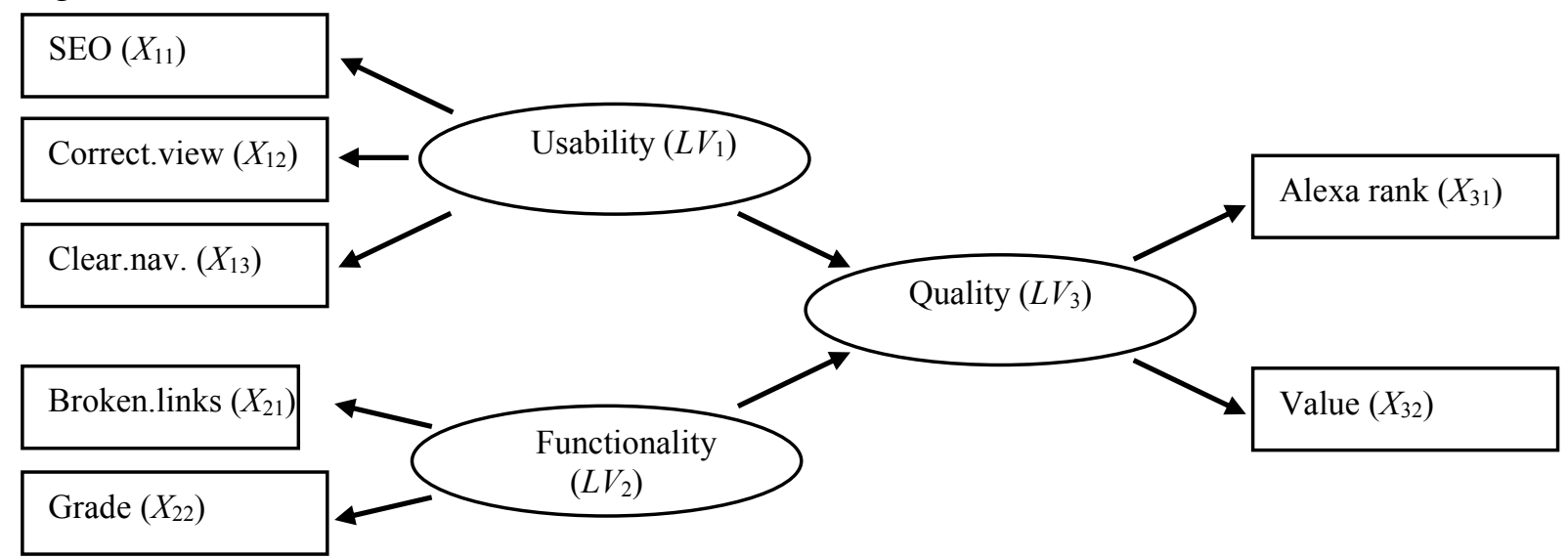

Source: Adopted from Král' and Laco (2018)

Manifest variables are represented in a rectangular form, latent variables are represented in an elliptical form and relationships between variables are represented with straight arrows.

The inner model consists of three latent variables that cannot be measured directly and relationships among them. We assume that overall quality of website is based on usability and functionality.

The outer model consists of relationships between individual latent variables and manifest variables they depend on.

Search Engine Optimization (in our model as SEO) is crucial for users to be able to find the corporate website on internet. SEO is a marketing discipline focused on growing visibility in organic (non-paid) search. According to Statcounter GlobalStat (gs.statcounter.com, 30-032019) the most searches Worldwide were $90 \%$ to $93 \%$ of all searches during last 12 months (in Slovakia it was 96\% to 98\%). There are several free on-line SEO Scoring Services. We used Sitechecker (sitechecker.pro). SEO rating may range from 0 (worst) to 100 (best).

Correct view of webpages (in our model as Correct.view) is another important aspect for web users. Especially today corporate websites are displayed on various devices with totally heterogeneous screen resolutions. That is why it is very important for corporate websites to be displayed correctly at various screen resolutions. In our model we evaluated this factor from scale 0 to 3 . Value 0 (worst) was assigned for websites that were displayed correctly only at traditional desktop screen resolution and 3 (best) was assigned for websites that were displayed correctly at all screen resolutions, that are used according to Statcounter GlobalStat (gs.statcounter.com, 10.5.2018). Value 2 was assigned to websites with some minor errors at some resolutions and value 1 was assigned to websites with major errors at most resolutions. 
Clear navigation (in our model as Clear.nav.) is important for users that came to corporate website. In our model we evaluated this factor from scale 0 (worst) to 3 (best). We considered if there is a main navigation available (at visible part of website in browser), if it is clear where in the structure of a website is a specific webpage, if there is a site search available.

As for the functionality, in our original model we assumed that it depends on the fact whether web standards are followed and speed of website. Originally, we measured the level of web standards followed by website as a number of errors and warnings (in our model as Error.warnings.) of HTML validator (http://validator.w3.org). We recognized that user experience might not be always affected by these standards, because it also depends on web browser that is used by web visitors. We also realized that larger website (containing large number of webpages) might contain larger number of errors. That is why we decided to use relative number - the ratio of broken (not working) URL links to the total number of URL links on all pages of the website. We used Site Checker: Free Broken Link Tool (http:// www.deadlinkchecker.com) for measurement. Verification of links contains hyperlinks, images and other HTML elements (details available at http://www.deadlinkchecker.com/ features.asp).

The speed of website was measured by Pingdom Website Speed Test (http://tools.pingdom.com). To achieve objectivity, we used the Google PageSpeed performance grade (in our model as Grade), which may range from 0 (worst) to 100 (best). It is described in more details at http://developers.google.com/speed.

The overall quality of website was measured by Alexa Traffic Rank and estimated value of the website. Alexa Traffic Rank (in our model as Alexa rank) is an estimate of the site's popularity. It is calculated using a combination of average daily visitors and pageviews of specific website over the past 3 months. We gained data for our research from http://www.alexa.com/siteinfo. The estimated value of the websites was gained from http://worthofweb.com.

We decided to investigate websites of top 250 manufacturing companies according to sales. Measurement of individual values of all parameters of all 250 websites was extremely time-consuming, as some measurements like broken links analysis took from several minutes to several hours depending on number of pages of the specific website. We were able to gather data for 189 websites. As for the companies that were excluded from research, most of them do not have a website at all. In 29 cases Alexa Traffic Rank could not be calculated due to low website traffic. In one case broken link analysis could not be performed due to site settings by company administrators.

The complete dataset is available at http://www.ef.umb.sk/amse2019. For modeling purposes, we reverted order of variables Grade and Broken.links. The corresponding correlation matrix containing Pearson correlation coefficients is listed in Table 1.

Table 1: Correlation matrix of manifest variables

\begin{tabular}{lccccccc}
\hline & SEO & Broken.links & Grade & Correct.view & Clear.nav. & Alexa rank & Value \\
\hline SEO & 1.00 & 0.07 & -0.05 & 0.09 & 0.09 & 0.11 & 0.06 \\
Broken.links & 0.07 & 1.00 & 0.15 & -0.04 & -0.05 & 0.15 & 0.01 \\
Grade & -0.05 & 0.15 & 1.00 & 0.00 & 0.00 & 0.21 & 0.23 \\
Correct.view & 0.09 & -0.04 & 0.00 & 1.00 & 0.36 & 0.24 & -0.05 \\
Clear.nav. & 0.09 & -0.05 & 0.00 & 0.36 & 1.00 & 0.28 & 0.07 \\
Alexa rank & 0.11 & 0.15 & 0.21 & 0.24 & 0.28 & 1.00 & 0.09 \\
Value & 0.06 & 0.01 & 0.23 & -0.05 & 0.07 & 0.09 & 1.00 \\
\hline
\end{tabular}

Source: the authors.

\subsection{Software}

We used statistical system R 3.5.1 (R Core Team, 2018) and R package plspm (Sanchez et al., 2017) for fitting models. 


\section{Results}

Our model is summarized in Tables $2-7$. Table 2 lists relationships between latent variables assumed in our model - usability, functionality, quality. We can see that there is a weak positive relationship between usability and quality of a web page and a very strong positive relationship between functionality and quality, respectively. We assume that such a large difference between these values is due to the nature of the chosen type of companies. Unlike retail businesses, in case of manufacturing companies website usability does not influence the overall quality of the website. On this basis we assume that in this case website usability is not so important for website visitors.

Table 2: Total effects of latent variables

\begin{tabular}{lccc}
\hline Relationships & Direct & Indirect & Total \\
\hline usability $\rightarrow$ functionality & 0.000 & 0 & 0.000 \\
usability $\rightarrow$ quality & 0.113 & 0 & 0.113 \\
functionality $\rightarrow$ quality & 0.900 & 0 & 0.900 \\
\hline
\end{tabular}

Source: the authors.

Values of Cronbach's reliability coefficient much smaller than 0.7 indicate that neither of latent variables (Usability, Functionality and Quality) could not be reliably measured. However, values of Dillon-Goldstein's rho statistics are greater than 0.7 for each of the blocks, i.e. each block is a good representation of it corresponding latent variable (see Table 3). According Sanchez (2013) "This index is considered to be a better indicator than the Cronbach's alpha because it takes into account to which extent the latent variable explains its block of indicators". Having the first eigenvalues bigger than one and the second smaller than one for usability is consistent with unidimensionality. On the other hand, for Functionality and Quality these values are quite close to one indicating that the corresponding manifest variables might be too weakly correlated to assume unidimensionality.

Table 3: Unidimensionality of model blocks

\begin{tabular}{lcccccc}
\hline \multicolumn{1}{c}{ Block } & Mode $^{*}$ & MVs $^{* *}$ & C.alpha & DG.rho & Eig.1st & Eig.2nd \\
\hline Usability & $\mathrm{A}$ & 3 & 0.396 & 0.703 & 1.40 & 0.963 \\
Functionality & $\mathrm{A}$ & 2 & 0.256 & 0.729 & 1.15 & 0.853 \\
Quality & $\mathrm{A}$ & 2 & 0.169 & 0.706 & 1.09 & 0.908 \\
\hline
\end{tabular}

Notes: ${ }^{*}$ Mode A means that a latent variable is in a reflective mode, i.e., the latent variable is seen as the cause of the manifest variables. ${ }^{* *} \mathrm{MVs}=$ The number of manifest variables corresponding to a latent variable.

Source: the authors.

Values of loadings $\left(\lambda_{j k}\right)$ (see Table 4) are satisfactory, i.e. greater or equal to 0.7 , for the variables Grade, Alexa rank and Value indicating a close relationship between individual variables and their corresponding latent variables. Less satisfactory are loadings of variables SEO, Correct.view and Clear.nav. and Error.warnings. Comunality $\left(\lambda_{j k}^{2}\right)$ shows the worst representation of the variable Broken.link as only $0.1 \%$ of its variability can be explained by variability of the latent variable quality. It indicates that our model may be omitting some key factors crucial for determining the financial value of a website.

Redundancy (computed as $\lambda_{j k}^{2} \cdot R^{2}$ ) measures fraction of the variance of an indicator in an endogenous block that is predicted by the independent latent variables associated to that block, i.e. functionality and usability predict about $77 \%$ variability of Alexa rank and about $71 \%$ of Value. By averaging redundancies of indicators, we get a mean redundancy which represents fraction of overall variability of indicators explained by independent latent variables (see Table 7). 
Table 4: Outer model

\begin{tabular}{lcccc}
\hline \multicolumn{1}{c}{ Block } & Weight & $\lambda_{j k}$ & Communality & Redundancy \\
\hline Usability & & & & \\
$\quad$ SEO & 0.567 & 0.662 & 0.438 & - \\
$\quad$ Correct.view & 0.453 & 0.675 & 0.456 & - \\
$\quad$ Clear.nav. & 0.469 & 0.679 & 0.460 & - \\
Functionality & & & & \\
$\quad$ Broken.links & 0.021 & 0.036 & 0.001 & - \\
$\quad$ Grade & 1.000 & 1.000 & 1.000 & - \\
Quality & & & & 0.772 \\
$\quad$ Alexa rank & 0.609 & 0.953 & 0.909 & 0.712 \\
$\quad$ Value & 0.458 & 0.916 & 0.838 & \\
\hline
\end{tabular}

Source: the authors.

Table 5 allows us to compare loadings of indicators and their cross-loadings, i.e. correlations with latent variables not corresponding to them. It indicates that all our exogenous variables are correctly assigned to blocks corresponding to respective latent variables and none of them should be reassigned to other block of variables.

Table 5: Loading and cross-loadings

\begin{tabular}{lccc}
\hline \multicolumn{1}{c}{ Block } & Usability & Functionality & Quality \\
\hline Usability & & & \\
$\quad$ SEO & 0.662 & 0.140 & 0.174 \\
Correct.view & 0.675 & 0.040 & 0.139 \\
$\quad$ Clear.nav. & 0.679 & 0.069 & 0.144 \\
Functionality & & & \\
$\quad$ Broken.links & 0.004 & 0.036 & 0.019 \\
$\quad$ Grade & 0.130 & 1.000 & 0.915 \\
Quality & & & 0.953 \\
$\quad$ Alexa rank & 0.286 & 0.915 & 0.916 \\
$\quad$ Value & 0.121 & 0.781 & \\
\hline
\end{tabular}

Source: the authors.

As we can see in Table 6, both Usability and Functionality have statistically significant coefficients. The pseudo goodness-of -fit statistics takes an acceptable value 0.705 . Overall (see Table 7), $85 \%$ of variability of the latent variable Quality was explained by our model, $87 \%$ of variability of the corresponding block are explained by the latent variable Quality and $74 \%$ of variability of indicators in an endogenous block is explained by usability and functionality.

Table 6: Coefficients of the inner model

\begin{tabular}{lcccc}
\hline \multicolumn{1}{c}{ Parameter } & Estimate $\left(\hat{\beta}_{j i}\right)$ & $\mathrm{SE}$ & $\mathrm{t}$-value \\
\hline Usability & 0.113 & 0.0287 & 3.94 & 0.0001 \\
Functionality & 0.900 & 0.0287 & 31.30 & 0.0000 \\
\hline
\end{tabular}

Source: the authors.

Table 7: Summary of inner model

\begin{tabular}{lcccc}
\hline \multicolumn{1}{c}{ Variable } & Type & $\mathrm{R}^{2}$ & Block communality & $\begin{array}{c}\text { Mean } \\
\text { redundancy }\end{array}$ \\
\hline Usability & Exogenous & - & 0.451 & - \\
Functionality & Exogenous & - & 0.500 & - \\
Quality & Endogenous & 0.849 & 0.873 & 0.742 \\
\hline
\end{tabular}

Source: the authors. 
Using bootstrap with 200 resamples of our sample websites, we got the following bootstrap confidence intervals for model parameters presented in the above tables (see Table 8).

\section{Conclusion}

Using PLS Path modelling we fitted a simple model generating latent variable scores for corporate websites. The model consists of three latent variables, namely Usability, Functionality and Quality of a website and seven reflective (exogenous) directly measurable variables (SEO, Correct.view, Clear.nav., Broken.links, Grade, Alexa rank, Value). We examined importance, through loading and weights, of particular variables in the corporate website evaluation process via loadings and weights. The generated scores allow us to rank corporate websites based on their usability, functionality and overall quality. We applied also bootstrapping here to get some further insight regarding the presented results but without intention to make inferential statements outside the sample at hand. Nevertheless, our analysis revealed that the ability of the selected variables to determine a value of was quite good given the simplistic model we used. From the point of view of applications, it is a promising result, as many other factors recommended in literature are difficult to measure. In our future research we plan to search for possibilities of website quality measurement automatization. In optimal case, we want to prepare online tool that could be easily used for this purpose.

Table 8: Validation via $95 \%$ two-sided bootstrap confidence intervals

\begin{tabular}{|c|c|c|c|c|}
\hline Measure & Original & Mean.Boot & LB & UB \\
\hline \multicolumn{5}{|l|}{ Weights } \\
\hline SEO & 0.567 & 0.358 & -0.578 & 0.782 \\
\hline Correct.view & 0.453 & 0.171 & -0.767 & 0.644 \\
\hline Clear.nav.* & 0.469 & 0.422 & 0.188 & 0.629 \\
\hline Broken.links & 0.021 & 0.080 & -0.011 & 0.551 \\
\hline Grade* & 1.000 & 0.975 & 0.591 & 1.000 \\
\hline Alexa rank ${ }^{*}$ & 0.609 & 0.615 & 0.501 & 0.919 \\
\hline Value* & 0.458 & 0.437 & 0.195 & 0.499 \\
\hline \multicolumn{5}{|l|}{ Loadings } \\
\hline SEO & 0.662 & 0.416 & -0.681 & 0.790 \\
\hline Correct.view & 0.675 & 0.352 & -0.744 & 0.856 \\
\hline Clear.nav. & 0.679 & 0.560 & -0.030 & 0.865 \\
\hline Broken.links* & 0.036 & 0.119 & 0.010 & 0.817 \\
\hline Grade* & 1.000 & 0.987 & 0.868 & 1.000 \\
\hline Alexa rank ${ }^{*}$ & 0.953 & 0.955 & 0.943 & 0.999 \\
\hline Value ${ }^{*}$ & 0.916 & 0.876 & 0.391 & 0.999 \\
\hline \multicolumn{5}{|l|}{ Paths } \\
\hline Usability $\rightarrow$ Quality & 0.113 & 0.163 & 0.000 & 0.514 \\
\hline Functionality $\rightarrow$ Quality* & 0.900 & 0.794 & 0.350 & 0.999 \\
\hline
\end{tabular}

Note: The symbol * denotes significant weights, loadings or paths.

Source: the authors.

\section{Acknowledgements}

Pavol Král' was supported by the grant scheme VEGA No. 1/0767/18 "SMART model - a decision support tool in management of enterprises" and Peter Laco was supported by the grant scheme KEGA No. 018UMB-4/2018 "Couching approach as a new form of critical thinking development of students in higher education". 


\section{References}

[1] Alva, M., Martinez, A., Cueva, J. 2003. Comparison of methods and existing tools for the measurement of usability in the web. In Levelle, J. M. C. et al. (eds.) Web Engineering ICWE 2003. Berlin : Springer, 2003, pp. 386-389.

[2] Chiou, C., Lin, C., Perng, C. 2010. A strategic framework for website evaluation based on a review of the literature from 1995 - 2006. In Information \& Management, 2010, vol. 47, iss. 5-6, pp. 282-290.

[3] Kollár, I., Král', P., Laco, P. 2014. Methodology for asessing website improvement in corporate environment. In Conference Proceedings of 17th International Scientific Conference Applications of Mathematics and Statistics in Economics. Wrocław : Wroclaw University of Economics, 2014, pp. 131-140. ISBN 978-83-7695-421-9.

[4] Kollár, I., Král', P., Laco, P. 2015. Measuring the level of corporate internet presentation in relation to economic performance. In Conference Proceedings of 18th International Scientific Conference Applications of Mathematics and Statistics in Economics. Prague : University of Economics, 2015. ISBN 978-80-245-2099-5.

[5] Král', P., Laco, P. 2018. Scoring of corporate websites using partial least squares path modelling. In Conference Proceedings of the 21st International Scientific Conference Applications of Mathematics and Statistics in Economics. Prague : University of Economics in Prague, 2018. ISBN 978-80-245-2277-7.

[6] Laco, P., Kallová, M. 2017. Factors influencing corporate websites' value. In Conference Proceedings of 20th International Scientific Conference Applications of Mathematics and Statistics in Economics. Wrocław : Wroclaw University of Economics, 2017. ISBN 978-83-7695-693-0.

[7] Lesáková, L. 2008. Influence of globalisation on small and medium enterprises. In Ekonomický časopis, 2008, vol. 56, iss. 6, pp. 607-621.

[8] Leporini, B., Paterno, F. 2003. Criteria for usability of accessible web sites. In Carbonell, N., Stephanidis, C. (eds.) U14ALL: Universal Access: Theoretical Perspectives, Practice, and Experience. Berlin : Springer, 2008, pp. 43-55.

[9] Palmer, J. W. 2002. Web site usability, design, and performance metrics. In Information Systems Research, 2002, vol. 13, iss. 2, pp. 151-167.

[10]R Core Team 2018: R: A language and environment for statistical computing. Vienna: R Foundation for Statistical Computing, 2018, http://www.r-project.org/.

[11]Robbins, S., Stylianou, A. 2003. Global corporate web sites: An empirical investigation of content and design. In Information \& Management, 2003, vol. 40, iss. 3, pp. 205-212.

[12] Sanchez, G. 2013. PLS Path Modeling with R Trowchez Editions. Berkeley. 222 pp. http://www.gastonsanchez.com/PLS Path Modeling with R.pdf.

[13] Sanchez, G., Trinchera, L., Russolillor, G. 2017. plspm: Tools for partial least squares path modeling (PLS-PM). R package, version 0.4.9, http://cran.r-project.org/web/ packages/plspm/index.html.

[14] Tezza, R., Bornia, A., de Andrade, D. 2011. Measuring web usability using item response theory: Principles, features and opportunities. In Interacting with Computers, 2011, vol. 23 , iss. 2, pp. 167-175. 